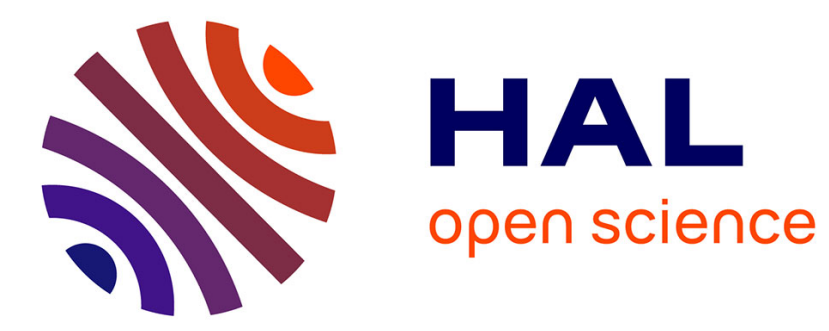

\title{
Phonon-Assisted Tunneling in High-Spin Molecules: Experimental Evidence
}

\author{
G Bellessa, N. Vernier, B. Barbara, D. Gatteschi
}

\section{To cite this version:}

G Bellessa, N. Vernier, B. Barbara, D. Gatteschi. Phonon-Assisted Tunneling in High-Spin Molecules: Experimental Evidence. Physical Review Letters, 1999, 83 (2), pp.416. hal-01660161

\section{HAL Id: hal-01660161 https://hal.science/hal-01660161}

Submitted on 10 Dec 2017

HAL is a multi-disciplinary open access archive for the deposit and dissemination of scientific research documents, whether they are published or not. The documents may come from teaching and research institutions in France or abroad, or from public or private research centers.
L'archive ouverte pluridisciplinaire HAL, est destinée au dépôt et à la diffusion de documents scientifiques de niveau recherche, publiés ou non, émanant des établissements d'enseignement et de recherche français ou étrangers, des laboratoires publics ou privés. 


\title{
Phonon-Assisted Tunneling in High-Spin Molecules: Experimental Evidence
}

\author{
G. Bellessa, ${ }^{1, *}$ N. Vernier, ${ }^{1}$ B. Barbara ${ }^{2}$ and D. Gatteschi ${ }^{3}$ \\ ${ }^{1}$ Laboratoire de Physique des Solides, Université Paris-Sud, Bâtiment 510, 91405 Orsay, France \\ ${ }^{2}$ Laboratoire de Magnétisme Louis Néel, CNRS, BP 166, 38042 Grenoble, France \\ ${ }^{3}$ Dipartimento di Chimica, Università di Firenze, Via Maragliano 77, 50144 Firenze, Italia
}

(Received 20 January 1999)

\begin{abstract}
We report on the observation of quantum tunneling in $\mathrm{Mn}_{12}$-acetate single crystals at a high frequency $(680 \mathrm{MHz})$ and low temperature (down to $20 \mathrm{mK}$ ). A peak in the imaginary part of the ac susceptibility as a function of magnetic field is observed. It arises from transitions between the two states of the fundamental doublet of the high-spin molecule. The tunneling rate is increased by setting the magnetic field perpendicular to the easy axis of magnetization of the crystals. Below $0.1 \mathrm{~K}$ the peak decreases strongly down to $0.02 \mathrm{~K}$. This effect is explained with a phonon-induced tunneling process.
\end{abstract}

PACS numbers: 75.45. $+\mathrm{j}, 63.20 .-\mathrm{e}, 72.10 . \mathrm{Di}$

Quantum tunneling of magnetization has been observed in high-spin $\mathrm{Mn}_{12}$-acetate $\left(\mathrm{Mn}_{12}\right.$-ac) molecules. It appears in steps in the hysteresis loop of oriented $\mathrm{Mn}_{12}$-ac crystals $[1,2]$. These experiments have been done with the magnetic field parallel to the easy axis of magnetization of the molecules, and thermally assisted tunneling has been proposed to explain the effect. However, this process cannot account for the effect at low temperature because the transverse magnetic field is too small $[3,4]$. The high-spin molecules have a fundamental doublet which is the only one populated at low temperature. Each state of this doublet is in a double-well potential with a high energy barrier (the anisotropy energy). Setting a magnetic field perpendicular to the easy axis of magnetization of the molecules increases the tunneling rate between the two states of the fundamental doublet $[5,6]$. Such an experimental setup allows one to study the tunneling across the energy barrier. It has been used to study the magnetic susceptibility at high frequency in glasses doped with rare-earth ions $(S=15 / 2)$ [7], in high-spin clusters $\mathrm{CrNi}_{6}(S=15 / 2)$ [8], and more recently in high-spin clusters $\mathrm{Fe}_{8}(S=10)$ [9]. In this Letter, we report on the observation of quantum tunneling in $\mathrm{Mn}_{12}$-ac molecules submitted to a magnetic field perpendicular to their anisotropy axis. A peak in the imaginary part of the ac susceptibility (at $680 \mathrm{MHz}$ ) as a function of magnetic field is observed at low temperature and decreases strongly below $0.1 \mathrm{~K}$ down to $0.02 \mathrm{~K}$. This new effect is explained with a phonon-induced tunneling process.

The crystals of $\mathrm{Mn}_{12}$-ac are built of discrete dodecanuclear $\mathrm{Mn}_{12}$ molecules, water of crystallization, and disordered acetic acid molecules [10]. The nominal composition is $\left[\mathrm{Mn}_{12}\left(\mathrm{CH}_{3} \mathrm{COO}\right)_{16}\left(\mathrm{H}_{2} \mathrm{O}\right)_{4} \mathrm{O}_{12}\right] \cdot 2 \mathrm{CH}_{3} \mathrm{COOH}$. $4 \mathrm{H}_{2} \mathrm{O}$. The crystal has a tetragonal symmetry, and the 12 manganese ions of each unit cell form a cluster with four inner $\mathrm{Mn}^{4+}(S=3 / 2)$ surrounded by height $\mathrm{Mn}^{3+}$ $(S=2)$. Exchange interactions inside the cluster stabilize a ferrimagnetic cluster ground state of spin $S=10$. A strong anisotropy energy of uniaxial symmetry is induced by the crystalline field [10]. The $\mathrm{Mn}_{12}$-ac single crystals are far from perfect. They have a mosaic structure, and the individual blocks of the mosaic are disoriented with respect to one another within about $1^{\circ}$.

The magnetic susceptibility was measured at high frequency using a split-ring resonator also called loop-gap resonator [11]. Its resonance frequency was around $680 \mathrm{MHz}$ and its quality factor was 3100 at low temperature. Because the electric field exists in the gap and not in the loop, this resonator is particularly suitable for magnetic susceptibility measurements. About 150 needleshaped single crystals (about $0.7 \mathrm{~mm}$ long and $0.2 \mathrm{~mm}$ in diameter) were glued on two faces of a silica cube with their easy axis of magnetization parallel between each other. The cube was put inside the ring where the alternating magnetic field irradiated the $\mathrm{Mn}_{12}$-ac crystals. The resonator was pressed against the wall of the mixing chamber of a ${ }^{3} \mathrm{He}-{ }^{4} \mathrm{He}$ dilution refrigerator. The static magnetic field was obtained from a superconducting magnet. It was applied perpendicularly to the easy axis of magnetization of the crystals, whereas the alternating magnetic field was parallel to the latter. To measure the magnetic susceptibility $\chi$, the resonance line of the resonator with the sample inside was determined using electromagnetic pulses at a low repetition rate (to avoid heating of the sample). The line shape was Lorentzian. The line broadening was proportional to the imaginary part of the susceptibility ( $\left.\chi^{\prime \prime}\right)$.

Figure 1 shows the variation of $\chi^{\prime \prime}$ as a function of magnetic field at $0.095 \mathrm{~K}$. There is a peak at $H=$ $6.06 \mathrm{~T}$. The peak height varies with temperature. Figure 2 shows this variation. There is a maximum around $0.1 \mathrm{~K}$, and remarkably a strong decrease of $\chi^{\prime \prime}$ with decreasing temperature below $0.1 \mathrm{~K}$.

We can explain the experimental peak as a function of magnetic field by starting from the Hamiltonian of a $\mathrm{Mn}_{12}$ cluster in an external magnetic field perpendicular to the easy axis of magnetization [5,6]:

$$
\mathcal{H}=-D S_{z}^{2}-g \mu_{B} \mathbf{H} \cdot \mathbf{S}
$$




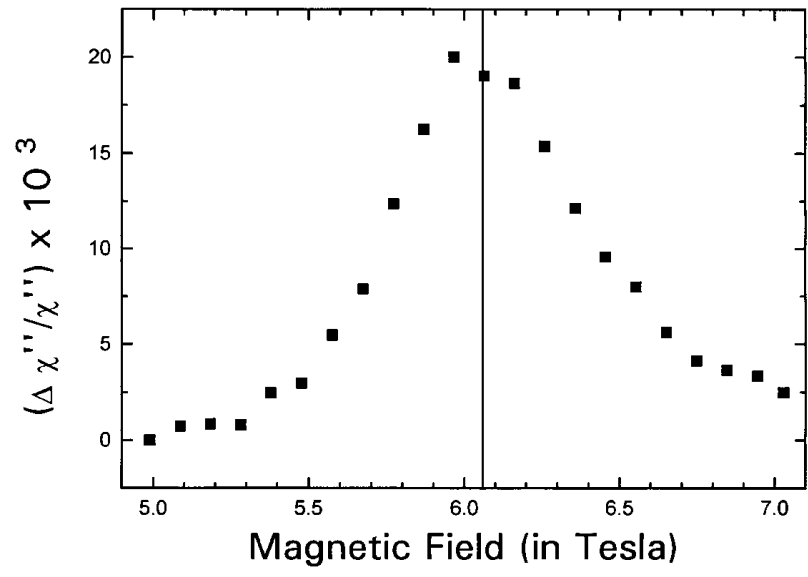

FIG. 1. Variation of the imaginary part of the ac susceptibility of $\mathrm{Mn}_{12}-\mathrm{ac}$ as a function of magnetic field at $680 \mathrm{MHz}$ and $0.095 \mathrm{~K}$. The magnetic field is perpendicular to the easy axis of magnetization of the crystals. The vertical line indicates the peak location obtained from the Hamiltonian using the parameters $D=0.68 \mathrm{~K}$ and $g_{\perp}=1.9$.

where $O z$ is the easy axis of magnetization, and $D$ is the anisotropy energy. We have not written in Eq. (1) the term $B_{4}^{4}\left(S_{+}^{4}+S_{-}^{4}\right)$ considered in Ref. [3] because it is quite small $\left(B_{4}^{4}=6 \times 10^{-5} \mathrm{~K}[12]\right)$. This term is needed in zero field to explain tunneling between the states $|-m\rangle$ and $|+m\rangle$. Here, we are in the configuration $H$ perpendicular to the easy axis of magnetization. In this case, the term $S_{x} H$ in Eq. (1) which does not commute with $S_{z}$ induces also tunneling between the states $|-m\rangle$ and $|+m\rangle$ [5], and prevails over the fourth-order term mentioned above. Since we can always choose the magnetic field in the $x \mathrm{Oz}$ plane, Eq. (1) can be written as

$$
\mathcal{H}=-D S_{z}^{2}-\frac{1}{2} g_{\perp} \mu_{B} H\left(S_{+}+S_{-}\right) .
$$

Numerical diagonalization of Eq. (2) with $D=0.68 \mathrm{~K}$ [13] and $g_{\perp}=1.9$ [12] gives the splitting of the two lowest states which depends on the magnetic field. For a splitting equal to the resonator energy $\hbar \omega$ (where $\omega$ is the angular frequency equal to $2 \pi \times 680 \mathrm{MHz}$ in our experiment) we obtain $H=6.06 \mathrm{~T}$ (the vertical line in Fig. 1), which fits perfectly the location of the susceptibility peak. The two states between which we observe transitions are no longer the states $|+10\rangle$ and $|-10\rangle$ but are mixings of the 21 states $|m\rangle$ of the cluster. These two lowest states $|+\psi\rangle$ and $|-\psi\rangle$ are, respectively, symmetrical and antisymmetrical [as it can be seen by calculating numerically the eigenvectors of Eq. (2)]. Here we observe transitions at a rate much higher than in the experiments of magnetization reversal [1,2]. The tunneling rate is increased by putting a high magnetic field perpendicular to the easy axis of magnetization of the $\mathrm{Mn}_{12}$ clusters, and thus lowering the energy barrier of the double-well potential $[5,6]$. This barrier $V$ can be evaluated classically. It is in the form $V=D S^{2}\left(1-g \mu_{B} H /\right.$

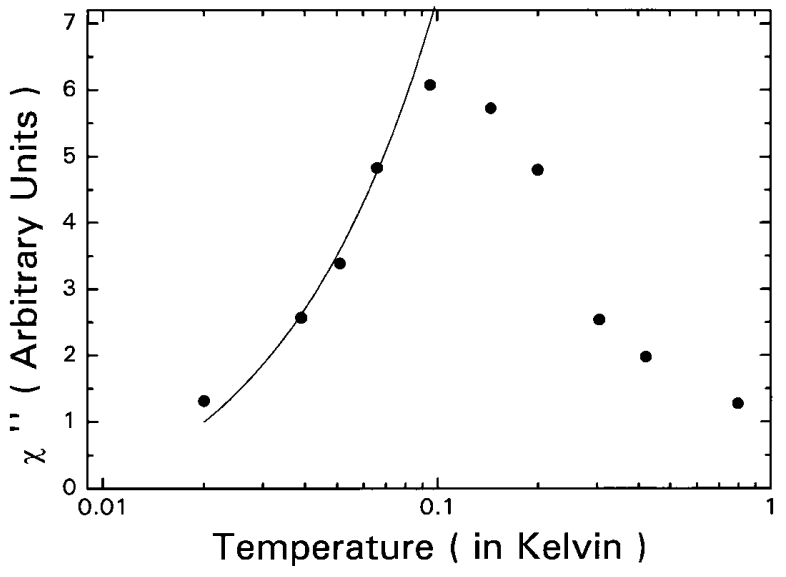

FIG. 2. Variation of the amplitude of the susceptibility peak as a function of temperature. The continuous line is obtained from Eq. (5).

$2 D S)^{2}$ and is equal to $13 \mathrm{~K}$ at $6 \mathrm{~T}$. So, it is much higher that the temperature of the experiment and the transitions between $|+\psi\rangle$ and $|-\psi\rangle$ occur by tunneling across the energy barrier.

We have explained the susceptibility peak with tunneling between the two lowest states $|+\psi\rangle$ and $|-\psi\rangle$ when the magnetic field is perpendicular to the easy axis. It is necessary to consider this last point, because it raises a question about the sample misalignment. Actually, the splitting of the two lowest states is of the form $\sqrt{\Delta^{2}+E_{z}^{2}}$, where $\Delta$ is the tunneling rate and $E_{z}$ is the Zeeman energy $2 g \mu_{B} H \cos (\theta)$ [5]. This term must be equal to our resonator energy $\hbar \omega(=0.032 \mathrm{~K})$. Hence, $E_{z}$ cannot exceed this value for $\Delta$ to be different from zero. To satisfy this condition $H$ must be perpendicular to the easy axis of the magnetic clusters within one-hundredth of a degree. Such an alignment is unfeasible experimentally. It is rather of the order of $1^{\circ}$. Nevertheless, we can explain the tunneling observation by taking into account the mosaic structure of the crystal. As seen above, the individual blocks of the mosaic are disoriented within $1^{\circ}$. So, we observe tunneling only in the blocks which have the good orientation. This explains why the amplitude of the signal from the resonator corresponds to a quite small part of the magnetic moments in the sample (of the order of $10^{-3}$ ).

The susceptibility peak as a function of magnetic field is well explained. Let us consider now its variation with temperature (Fig. 2). Above $0.1 \mathrm{~K}$, the amplitude of this peak decreases with increasing temperature and is quite small above $1 \mathrm{~K}$. This behavior has already been observed in high-spin susceptibility measurements [7-9]. Above $0.3 \mathrm{~K}$, it is very difficult to observe quantum transitions inside the fundamental doublet, presumably because the transverse relaxation time $T_{2}$ of these tunneling states becomes too small. The proof that the relaxation times $T_{1}$ and $T_{2}$ are very small is given by the fact that we do not observe either the nonlinear dependence of the 
susceptibility on the amplitude of the ac field as in Ref. [8] or tunneling-state echoes (the equivalent of spin echoes) as in high-spin rare-earth ions [14]. The ligand field is modulated by the lattice vibrations, and increasing the temperature can induce a decrease of the relaxation times.

The decrease of the peak amplitude below $0.1 \mathrm{~K}$ shown in Fig. 2 is a quite unusual effect. Generally, $\chi^{\prime \prime}$ increases with decreasing temperature and then becomes constant in the temperature range $2 k_{B} T \ll \hbar \omega$. Here we have a new effect which is an increasing tunneling rate with increasing temperature below $0.1 \mathrm{~K}$. We can explain this effect with phonon-assisted tunneling. A thermally assisted tunneling process different from the effect we propose here has been proposed to explain tunneling at higher temperature: It was a thermally activated process into high-lying excited states and then a tunneling process [3,15-17]. This effect cannot work at low temperature, since only the two lowest states are populated below $1 \mathrm{~K}$. We can explain our effect with a true phonon-induced tunneling: the tunneling rate between the two lowest states is indeed increased by the presence of resonant thermal phonons (their energy is equal to the splitting of the two states). The imaginary part of the ac susceptibility can be written as [18]

$$
\chi^{\prime \prime}=C N \Delta^{2} f(\omega) T_{2} \tanh \left(\frac{\hbar \omega}{2 k_{B} T}\right),
$$

where $C$ is a constant, $N$ is the number of spins, and $\Delta$ is the magnetic dipole matrix element between the two states of the fundamental doublet. The shape function $f(\omega)$ is a Lorentzian function of the applied frequency $\omega$, the resonance frequency $\omega_{0}$ (which depend on the applied magnetic field), and the relaxation time $T_{2}$ describing the linewidth. The last term of Eq. (3) is often approximated to $\hbar \omega / 2 k_{B} T$, but this cannot be done here because we are not in the temperature range $k_{B} T \gg \hbar \omega$. We explain our effect by assuming that the tunneling rate $\Delta$ in Eq. (3) is induced by a two-phonon process (the interaction mechanism being provided by the ligand field): the magnetic moment makes a transition from $|+\psi\rangle$ to $|-\psi\rangle$ (or vice versa) and absorbs (or emits) a quantum $\hbar \omega$ from the acmagnetic field only if a phonon of angular frequency $\omega$ is absorbed and then reemitted after the transition. This process is quite similar to a Raman process, except the frequencies of the two phonons are the same. It gives a matrix element for the transition which is multiplied by $[n(n+1)]^{1 / 2}$, where $n$ is the phonon occupation number $\left[\exp \left(\hbar \omega / k_{B} T\right)-1\right]^{-1}[19]$. Hence, Eq. (3) becomes

$$
\chi^{\prime \prime}=C N \Delta^{2} n(n+1) f(\omega) T_{2} \tanh \left(\frac{\hbar \omega}{2 k_{B} T}\right) .
$$

Giving an explicit form of $n$, we obtain for the amplitude of the susceptibility peak $[f(\omega)=1]$

$$
\chi^{\prime \prime}=2 C N \Delta^{2} T_{2} \sinh ^{-1}\left(\frac{\hbar \omega}{k_{B} T}\right) .
$$

This equation fits very well the experimental variation of $\chi^{\prime \prime}$ below $0.1 \mathrm{~K}$ (the continuous line in Fig. 2). Equation (5) gives a susceptibility which continues to increase above $0.1 \mathrm{~K}$. However, we do not observe such a variation because, as we have seen above, $T_{2}$ becomes too short and makes the susceptibility peak disappear.

We have considered above the tunneling enhanced by phonons, which is the predominant effect in our temperature range. However, the bare tunneling must exist. It gives a susceptibility $\chi^{\prime \prime}$ according to Eq. (3) [18], which corresponds to excitations by photons of transitions between the two states $|+\psi\rangle$ and $|-\psi\rangle$. This effect has been observed in rare-earth ions [7] and magnetic clusters [8,9]. It is perhaps this effect which is beginning to appear in Fig. 2 at our lowest temperature of $0.02 \mathrm{~K}$, where the experimental point is slightly above the predicted curve of phonon-induced tunneling. Below $0.02 \mathrm{~K}$, it is expected that with decreasing temperature $\chi^{\prime \prime}$ reaches a plateau corresponding to the bare tunneling rate.

To conclude, we have observed quantum tunneling in spin-10 $\mathrm{Mn}_{12}$ molecular clusters in transverse magnetic field at low temperature. We have shown that the ac-susceptibility peak is well explained by assuming transitions between the two lowest states of the fundamental doublet. Because of a high magnetic field perpendicular to the easy axis of magnetization the tunneling rate between these states is increased. Lastly, the transitions between these states are induced by a Ramanlike two-phonon process and reduce strongly down to $0.02 \mathrm{~K}$, which is an experimental evidence for phononassisted tunneling.

*Electronic address: bellessa@1ps.u-psud.fr

[1] J. R. Friedman, M. P. Sarachik, J. Tejada, and R. Ziolo, Phys. Rev. Lett. 76, 3830 (1996).

[2] L. Thomas, F. Lionti, R. Ballou, D. Gatteschi, R. Sessoli, and B. Barbara, Nature (London) 383, 145 (1996).

[3] P. Politi, A. Rettori, F. Hartmann-Boutron, and J. Villain, Phys. Rev. Lett. 75, 537 (1995).

[4] L. Gunther, Europhys. Lett. 39, 1 (1997).

[5] I. Ya. Korenblit and E. F. Shender, Sov. Phys. JETP 48, 937 (1978).

[6] D. A. Garanin and E. M. Chudnovsky, Phys. Rev. B 56, 11102 (1997).

[7] N. Vernier and G. Bellessa, Phys. Rev. Lett. 71, 4063 (1993).

[8] N. Vernier, G. Bellessa, T. Mallah, and M. Verdaguer, Phys. Rev. B 56, 75 (1997).

[9] E. del Barco, N. Vernier, J. M. Hernandez, J. Tejada, E. M. Chudnovsky, E. Molins, and G. Bellessa (to be published).

[10] T. Lis, Acta Crystallogr. B 36, 2042 (1980).

[11] W. N. Hardy and L. A. Whitehead, Rev. Sci. Instrum. 52, 213 (1981). 
[12] A. L. Barra, D. Gatteschi, and R. Sessoli, Phys. Rev. B 56, 8192 (1997).

[13] S. Hill, J. A. A. J. Perenboom, N. S. Dalal, T. Hathaway, T. Stalcup, and J.S. Brooks, Phys. Rev. Lett. 80, 2453 (1998).

[14] N. Vernier, G. Bellessa, and D. A. Parshin, Phys. Rev. Lett. 74, 3459 (1995).

[15] M. A. Novak and R. Sessoli, in Quantum Tunneling of Magnetization, edited by L. Gunther and B. Barbara (Kluwer Academic Publishers, Dordrecht, 1995), p. 171.
[16] F. Luis, J. Bartolomé, J.F. Fernandez, J. Tejada, J. M. Hernandez, X. X. Zhang, and R. Ziolo, Phys. Rev. B 55, 11448 (1997).

[17] B. Barbara, L. Thomas, F. Lionti, A. Sulpice, and A. Caneschi, J. Magn. Magn. Mater. 177-181, 1324 (1998).

[18] A. Abragam and B. Bleaney, Electron Paramagnetic Resonance of Transition Ions (Dover Publications, New York, 1986), p. 122.

[19] A. Abragam, Principles of Nuclear Magnetism (Oxford Science Publications, Oxford, 1996), p. 407. 